\title{
In-situ Polymerization of Carboxyl-functionalized Graphene Oxide and Polyaniline Composites for Supercapacitor
}

\author{
Zhao Xia Hou', a , Si Ming Li ${ }^{1}$, Lin Li ${ }^{1}$, Hai Bo Long ${ }^{1}$, Mei Han Wang ${ }^{1}$ \\ ${ }^{1}$ Liaoning Province Key Laboratory of New Functional Materials and Chemical Technology, School \\ of Mechanical Engineering, Shenyang University, Shenyang 110044, China \\ aluckyxia2007@126.com
}

\begin{abstract}
Keywords: Graphene oxide, Polyaniline, Composites, Carboxyl-functionallization, Electrochemical performance.

Abstract. Carboxyl-functionallized graphene oxide and polyaniline composites (FGO/PANI) were fabricated by in-situ polymerization method. The microstructure, morphology and electrochemical performance were characterized. Graphene oxide/polyaniline composites (GO/PANI) are short rod-like and agglomerate severely. PANI in FGO/PANI appeares long and thin rod and dispersed uniformly. Some of the $-\mathrm{C}-\mathrm{O}-\mathrm{C}-$ are converted to $-\mathrm{OH}$ by $\mathrm{HBr}$ and $-\mathrm{OH}$ connverts to $\mathrm{COOH}-$ by oxalic acid. The specific capacitance of FGO/PANI ( $297 \mathrm{~F} / \mathrm{g}$ ) is higher than GO/PANI (226 F/g). The charge transfer resistance of GO/PANI was reduced after carboxyl-functionallization.
\end{abstract}

\section{Introduction}

A large amount of research have been made on graphene-based or graphene-derived materials as electrodes in supercapacitors due to the reasonably high electrical conductivity[1-3]. Graphene oxide (GO) is of the similar structure with graphene, in addition, a lot of active functional groups are distributed in its surface, it is considered a functional graphene as an important derivative of traditional graphene. At present, many functional groups such as polylysine, amine, polyamide, porphyrin and fullerene have been grafted on graphene oxide or graphene. The grafting of these functional groups has made the application of graphene more widely, and carves out a new way for the development of future energy.

Carboxyl group, as an important functional one in organic synthesis, is a precursor of the preparation of many organics by the acylation, esterification and ammoniation. In the functionalization of GO, carboxyl groups can be used to further introduce the active groups of biomolecules, macromolecules and small molecules into graphene. It is found that, carboxyl is mainly in GO layer flat edge position[4], and the epoxy groups and hydroxyl mainly exist in the graphene oxide layer plane, most of the preparation of graphene/ polyaniline (PANI) composites[5-7] ignore the $-\mathrm{OH}$ and $-\mathrm{O}-$ in the plane, and just use the carboxyl in the edge of the lamella, this may cause PANI to move around the graphene sheet and result in incomplete and unstable composition. Adoption of a certain functional approach to the epoxy and hydroxyl groups in the plane of GO into carboxyl, then compositing with PANI, can make the PANI conductive polymers order and stable in the graphene surface.

\section{Experimental}

Chemicals. All reagents used in this work are analytical grade. Graphite powder were purchased from Tianjin Ruijinte Chemical Reagent Company. Aniline(An), oxalic acid, ammonium persulfate (APS), hydrobromic acid were purchased from Tianjin Bodi Chemical Engineering Corporation.

Synthesis of FGO. GO was prepared by a modyfied Hummers method. Tipically, HBr was added to $1 \mathrm{mg} / \mathrm{mL} \mathrm{GO}$ suspension with constant stirring at $90{ }^{\circ} \mathrm{C}$ for $12 \mathrm{~h} .1 .0 \mathrm{~g}, 1.5 \mathrm{~g}$ or $2.0 \mathrm{~g}$ oxalic acid were added to the mixture and continuously stirred at $90{ }^{\circ} \mathrm{C}$ for $4 \mathrm{~h}$, respectively. Suspension were centrifuged and washed to neutral, and then dried at $60{ }^{\circ} \mathrm{C}$ for $12 \mathrm{~h}$. Samples were marked as FGO ${ }_{1}$, $\mathrm{FGO}_{2}$ and $\mathrm{FGO}_{3}$, respectively . 
Synthesis of FGO/PANI composites. Tipically, APS and $\mathrm{FGO}_{3}$ were added to $100 \mathrm{~mL}$ of $1 \mathrm{~mol} / \mathrm{L}$ $\mathrm{HCl}$ and stirred for $2 \mathrm{~h}$, respectively. An was added to $\mathrm{FGO}_{3}$ suspension. And then, APS solution was droped to the $\mathrm{FGO}_{3}$ suspension with stirring and keep reacting for $0.5 \mathrm{~h}$. The resultant mixture solution was ultrasonicly treatment for $10 \mathrm{~min}$ and washed consecutively to neutral with deionized water. Finally, the $\mathrm{FGO}_{3} / \mathrm{PANI}$ composites were dried at $60{ }^{\circ} \mathrm{C}$ for $12 \mathrm{~h}$. Sample marked as $\mathrm{FGO}_{3} / \mathrm{PANI}$.

Preparation of the electrode. PANI, GO/PANI and $\mathrm{FGO}_{3} / \mathrm{PANI}$ acted as active materials to prepare the electrode, respectively. The mass ratio of active materials: binder (PTFE) is 90:10. The electrode dried at $120{ }^{\circ} \mathrm{C}$ for $12 \mathrm{~h}$. The two-electrod system was assemblied with a cellulose paper as separator, carbon fibre paper as current collector and $1 \mathrm{M} \mathrm{H}_{2} \mathrm{SO}_{4}$ aqueous as electrolyte.

Characterization. X-ray diffraction (XRD) was analyzed by a PW3040/60 X'Pert Pro MPD XRD (PANalytical) with $\mathrm{Cu} \mathrm{K \alpha}$ radiation $(\lambda=0.15444 \mathrm{~nm})$ at $40 \mathrm{kV}$ and $40 \mathrm{~mA}$ with $5^{\circ} / \mathrm{min}$ from $5^{\circ}$ to $80^{\circ}$. Foutier transform infrared spectrometer (FT-IR) was recorded with Bruker IFS 55 FT-IR spectrometer at room temperature from 400 to $4000 \mathrm{~cm}^{-1}$ at a resolution of $4 \mathrm{~cm}^{-1}, \mathrm{KBr}$ powder pellet. The microstructure was observed by an S4800 Scanning Electron Microscope (SEM, Hitachi, Tokyo, Japan). All the electrochemical performance was studied on a PARSTART 2237 electrochemical workstation(China). Cyclic voltammetry (CV) and electrochemical impedance spectroscopy (EIS ) were carried out in a two-electrod system with $1 \mathrm{M} \mathrm{H}_{2} \mathrm{SO}_{4}$ aqueous electrolyte. $\mathrm{CV}$ were conducted within the potential range of $0 \sim 0.8 \mathrm{~V}$ at a scan rate of 5, 10, 25, 50, 100 and $200 \mathrm{mV} / \mathrm{s}$. The frequency limits were typically set from $0.01 \mathrm{~Hz} 100 \mathrm{kHz}$ with a voltage amplitude of $5 \mathrm{mV}$.

\section{Results and Discussion}

Microstructure and morphology analysis. XRD patterns of graphite and GO are shown in Fig. 1 (a) and XRD patterns of PANI, GO/PANI and $\mathrm{FGO}_{3} / \mathrm{PANI}$ are shown in Fig. 1 (b). Graphite shows its characteristic peaks around $26.6^{\circ}$ and $54.5^{\circ}$, contributed to (002) and (004), respectively. A broad peak around $11^{\circ}$ and a weak peak around $43^{\circ}$ are observed in GO, contributed to (001) and (100). These peaks indicate that oxidative groups and $\mathrm{H}_{2} \mathrm{O}$ molecules have been inserted into the interlayer of the graphite.

Diffraction peaks around $25.4^{\circ}, 20.5^{\circ}$ and $14.9^{\circ}$ are corresponding to the characteristic peaks of PANI at (200), (020) and (011), respectively. The peaks of PANI are stronger than GO/PANI and $\mathrm{FGO}_{3} / \mathrm{PANI}$, which indicates the degree of structural order decreases after composition. While the degree of structural order of $\mathrm{FGO}_{3} / \mathrm{PANI}$ is better than GO/PANI. The diffration peaks of GO can not be observed in Fig. 1 (b), which is owing to that the weight of PANI acounts for $90 \%$ in the composites, the diffraction peaks of PANI are overwhelming.
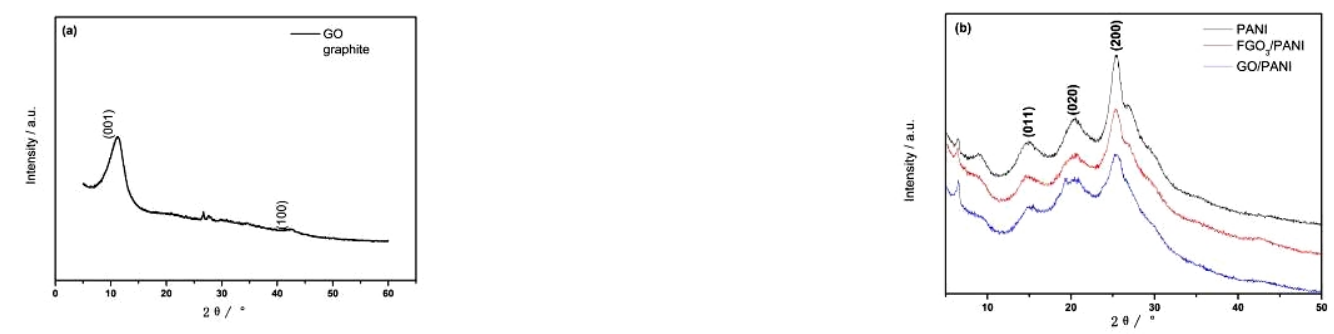

Fig. 1. XRD patterns of graphite, GO, PANI, GO/PANI and $\mathrm{FGO}_{3} / \mathrm{PANI}$
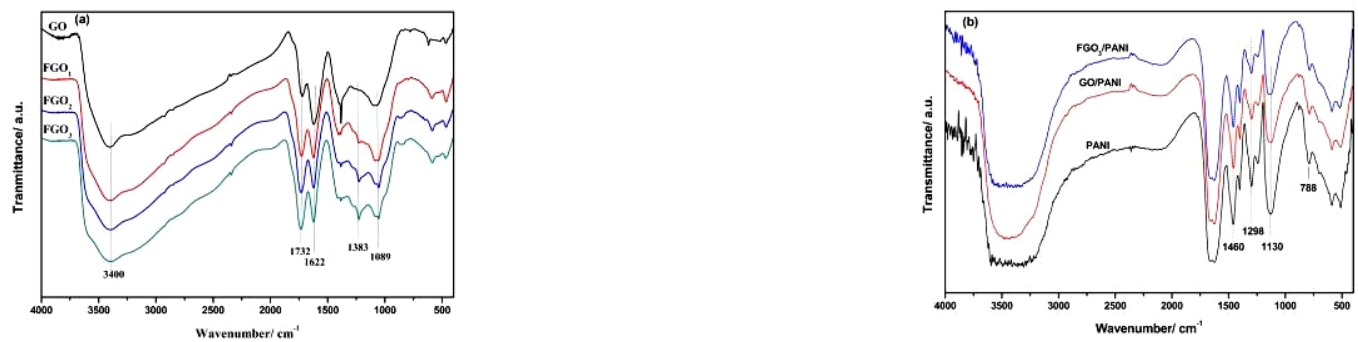

Fig. 2. FT-IR spectra of $\mathrm{GO}, \mathrm{FGO}_{1}, \mathrm{FGO}_{2}$ and $\mathrm{FGO}_{3}$ (a) PANI, GO/PANI and $\mathrm{FGO}_{3} / \mathrm{PANI}$ (b) 
Fig. 2 (a) shows the FTIR spectra of GO, $\mathrm{FGO}_{1}, \mathrm{FGO}_{2}$ and $\mathrm{FGO}_{3}$. The absorption peak at $1089 \mathrm{~cm}^{-1}$ is related to the stretching of $-\mathrm{C}-\mathrm{O}-\mathrm{C}-$. The absorption peak at $1383 \mathrm{~cm}^{-1}$ is related to the stretching of $\mathrm{C}-\mathrm{OH}$. The absorption peak at $1622 \mathrm{~cm}^{-1}$ is related to the bending of $\mathrm{C}=\mathrm{C}$. The absorption peak at $1732 \mathrm{~cm}^{-1}$ is related to the stretching of $\mathrm{C}=\mathrm{O}$ in $-\mathrm{COOH}$. The absorption peak at $3400 \mathrm{~cm}^{-1}$ is related to the stretching of $-\mathrm{OH}$. Compared with the ratio of peaks at $1732 \mathrm{~cm}^{-1}$ and $1622 \mathrm{~cm}^{-1}$, the absorption peaks at $1732 \mathrm{~cm}^{-1}$ intense obviously after carboxyl functionalization and increase with the content of oxalic acid. In addition, the absorption peaks at $1383 \mathrm{~cm}^{-1}$ and $1089 \mathrm{~cm}^{-1}$ are evidently decreased after functionalization. It is indicated that some of the $-\mathrm{C}-\mathrm{O}-\mathrm{C}-$ are converted to $-\mathrm{OH}$ by $\mathrm{HBr}$ and esterification reaction take place between oxalic acid and $-\mathrm{OH}$.

Fig. 2 (b) shows the FTIR spectra of PANI, GO/PANI and $\mathrm{FGO}_{3} / \mathrm{PANI}$. The three spectra are quite similar owing to the overwhelming majority of PANI in composites. The main characteristic peaks of PANI located at $1460 \mathrm{~cm}^{-1}, 1298 \mathrm{~cm}^{-1}, 1130 \mathrm{~cm}^{-1}$ and $788 \mathrm{~cm}^{-1}$ are assigned to $\mathrm{C}=\mathrm{C}$ strenching of the benzenoid rings, $\mathrm{C}-\mathrm{N}$ strenching of second amine, $\mathrm{C}=\mathrm{N}$ stretching of quinoid rings and $\mathrm{C}-\mathrm{H}$ bending of benzenoid rings, respectively. The broad intensive band from 1770 to $1530 \mathrm{~cm}^{-1}$ should be attributed to the absorption of $\mathrm{C}=\mathrm{C}$ stretching of the quinoid rings and $\mathrm{C}=\mathrm{C}$ bending of benzenoid rings. The broad intense band from $3600 \mathrm{~cm}^{-1}$ to $3200 \mathrm{~cm}^{-1}$ is assigned to the stretching of $-\mathrm{OH}$ and $-\mathrm{N}-\mathrm{H}$. In addition, under close observation, the ratio of the peak area at $1460 \mathrm{~cm}^{-1}, 1298 \mathrm{~cm}^{-1}$ and $1130 \mathrm{~cm}^{-1}$ are different, the maximum is PANI and the minimum is FGO/PANI.
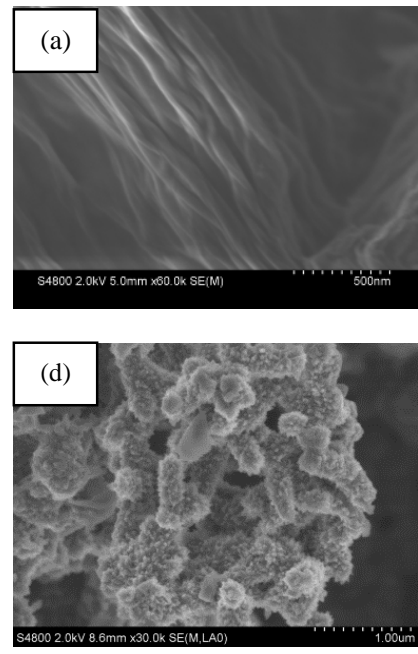
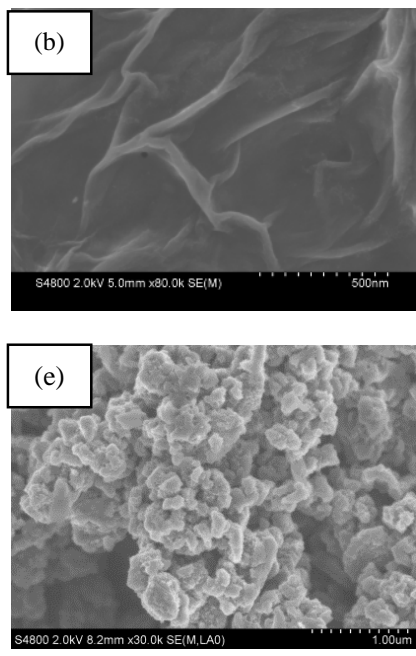
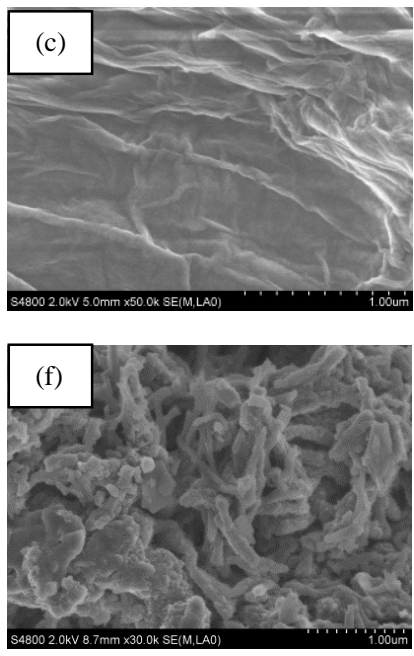

Fig. 3. SEM images of graphite oxide(a), $\mathrm{GO}(\mathrm{b}), \mathrm{FGO}_{3}(\mathrm{c}), \mathrm{PANI}(\mathrm{d}), \mathrm{GO} / \mathrm{PANI}(\mathrm{e})$ and $\mathrm{FGO}_{3} / \mathrm{PANI}(\mathrm{f})$

The SEM images of graphite oxide, $\mathrm{GO}, \mathrm{FGO}_{3}$, PANI, GO/PANI and $\mathrm{FGO}_{3} / \mathrm{PANI}$ are shown in Fig. 3. It can be seen that the stratification of graphite is obvious after chemical exfoliation and the layer is relatively thick. After ultrasonic treatment, corrugation can be observed on $\mathrm{GO}$ and $\mathrm{FGO}_{3} \mathrm{sample}$ Rod-like PANI with flocky feature is observed in Fig. 3 (d), and exists a little agglomeration. $\mathrm{GO} / \mathrm{PANI}$ appears short rod-like and severely agglomerated. PANI in $\mathrm{FGO}_{3} / \mathrm{PANI}$ appears relatively long and thin rod and disperses uniformly, and PANI growing on graphene oxide sheets can be observed in Fig. 3 (f). It is indicated that carboxyl functionalization is favourable to the composition of PANI and GO.
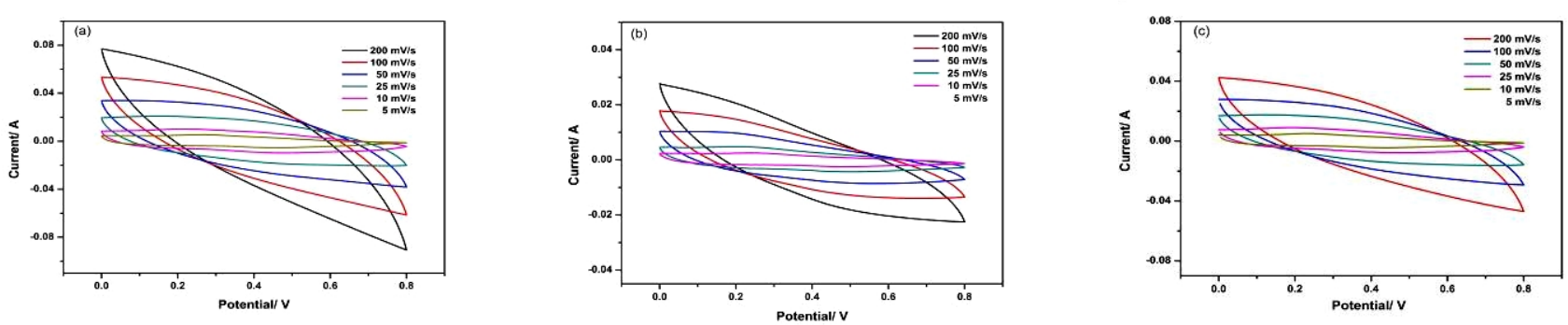

Fig. 4. $\mathrm{CV}$ curves of PANI (a), GO/PANI (b) and $\mathrm{FGO}_{3} / \mathrm{PANI}$ (c) at different scanning rate 
Electrochemical performance. $\mathrm{CV}$ curves of PANI , GO/PANI and $\mathrm{FGO}_{3} / \mathrm{PANI}$ at different scanning rate of $5 \mathrm{mV} / \mathrm{s}, 10 \mathrm{mV} / \mathrm{s}, 25 \mathrm{mV} / \mathrm{s}, 50 \mathrm{mV} / \mathrm{s}, 100 \mathrm{mV} / \mathrm{s}$ and $200 \mathrm{mV} / \mathrm{s}$ are shown in Fig. 4 (a), (b) and (c), respectively. Two pairs of redox peaks in the CV curves of PANI, GO/PANI and $\mathrm{FGO}_{3} / \mathrm{PANI}$ are contributed to the redox transition of leucoemeraldine/emeraldine/perniggraniline states of PANI. The gravimetric specific capacitance of the three supercapacitors at $5 \mathrm{mV} / \mathrm{s}$ were calculated from the $\mathrm{CV}$ curves in Fig. 4. The specific capacitance of $\mathrm{FGO}_{3} / \mathrm{PANI} （ 297 \mathrm{~F} / \mathrm{g}$ ） is higher than GO/PANI (226 F/g), which indicates that carboxyl functional GO is helpful to improve the hybrids capacitance. $\mathrm{FGO}_{3} / \mathrm{PANI}$ with the long rod structure without aggregation is better than GO/PANI with small rod aggregation in terms of specific capacitance. However, they are both lower than PANI ( $349 \mathrm{~F} / \mathrm{g}$ ), which is due to $\mathrm{GO}$ and $\mathrm{FGO}_{3}$ are not conductive.

Fig. 5 shows the Nyquist plots of PANI, GO/PANI and $\mathrm{FGO}_{3} / \mathrm{PANI}$ in the frequency range of $0.01-100 \mathrm{kHz}$ at the open circuit potential with expanded plots in the inset. The Nyquist plot of $\mathrm{FGO}_{3} / \mathrm{PANI}$ is similar to that of GO/PANI, but showing a smaller semicircle in the high-frequency region, which reveals a good capacitive behavior. The devices show relatively small equivalent series resistance $\left(R_{s}\right)$ values $(0.2 \sim 0.3 \Omega)$, indicating the lower internal resistance of the devices. The second intersection point of the semicircle on the real axis, represents the total resistance $\left(R_{c t}\right)$ at the interface between the elexctrode and electrolyte. It is easy to see that the $\mathrm{R}_{\mathrm{ct}}$ of $\mathrm{FGO}_{3} / \mathrm{PANI}$ is lower than that of GO/PANI, which indicates that the charge transfer resistance of the composites reduce after carboxyl-functionallization.

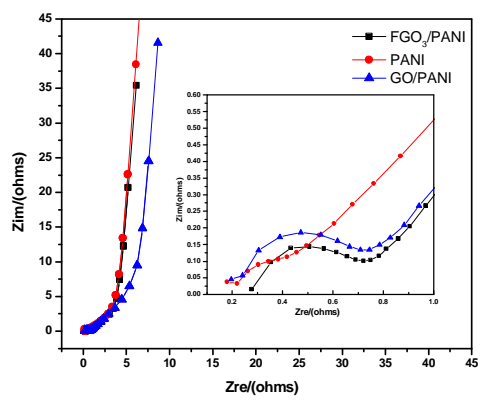

Fig. 5. Nyquist plots of PANI, GO/PANI and $\mathrm{FGO}_{3} / \mathrm{PANI}$

\section{Conclusions}

Carboxyl functional approach to the epoxy and hydroxyl groups in the plane of GO, then compositing with PANI, can make the PANI conductive polymers arrange orderly and uniform. GO/PANI is short rod-like and agglomerate. PANI in FGO /PANI appeares long and thin rod, and disperses uniformly. The degree of structural order of FGO/PANI is better than GO/PANI. The absorption peak at 1732 $\mathrm{cm}^{-1}(\mathrm{C}=\mathrm{O}$ in $-\mathrm{COOH})$ intenses obviously and the absorption peaks at $1383 \mathrm{~cm}^{-1}(\mathrm{C}-\mathrm{OH})$ and 1089 $\mathrm{cm}^{-1}$ (-C-O-C) decrease after carboxyl-functionalization. The specific capacitance of FGO/PANI ( 297 $\mathrm{F} / \mathrm{g}$ ) is higher than GO/PANI (226 F/g). The charge transfer resistance of composites reduces after carboxyl-functionallization.

\section{Acknowledgments}

This work was financially supported by the Program of Natural Science Foundation of China (No. 51472166) and Liaoning BaiQianWan Talents program.

\section{References}

[1] Z.J. Fan, Q.K. Zhao, T.Y. Li, J. Yan, Y.M. Ren, J. Feng, T. Wei:Carbon Vol. 5 (2012) , p. 1699

[2] Z.Q. Niu, J. Chen, H.H. Hng, J. Ma, X.D. Chen: Adv. Mater Vol. 24 (2012), p. 4144

[3] T. Kim, G. Jung, S. Yoo, K.S. Suh, R.S. Ruoff: ACS Nano Vol. 7 (2013), p. 6899 
[4] R. Yuge, M. Zhang, M. Tomonari, T. Yoshitake, S. Iijima: ACS Nano Vol.9 (2008), p. 1865

[5] B. Chitragara, W.J. Kim, Y.D. Kim, D.S. Huh: Materials Letters Vol. 19-20 (2011), p. 3120

[6] H. Wang, Q. Hao, X. Yang, L. lu: ACS Applied Materials \& Interfaces Vol 3 (2010), p. 821

[7] K. Zhang, L.L. Zhang, X. Zhao, J. Wu: Chemistry of Materials Vol 4 (2010), p. 1392 